\title{
Impact of dedicated women's outreach workers (WOWs) on recruitment of women in ACTG clinical studies
}

\author{
Elizabeth Barr ${ }^{1,2}$ (D), Karine Dubé ${ }^{3}$ (D), Shobha Swaminathan ${ }^{4}$, \\ Carlos Del Rio ${ }^{5}$ (D), Danielle M. Campbell ${ }^{6,7}$, Marta Paez-Quinde ${ }^{4}$ and \\ Susan E. Cohn ${ }^{8}$
}

\author{
${ }^{1}$ AIDS Clinical Trials Group (ACTG) Community Scientific Subcommittee (CSS), Baltimore, MD, USA; \\ ${ }^{2}$ Department of Gender, Women's, \& Sexuality Studies, UMBC, Baltimore, MD, USA; ${ }^{3}$ UNC Gillings School \\ of Global Public Health, Chapel Hill, NC, USA; ${ }^{4}$ Department of Medicine, Rutgers New Jersey Medical \\ School, Newark, NJ, USA; ${ }^{5}$ Department of Medicine, Division of Infectious Diseases, Emory University \\ School of Medicine, Atlanta, GA, USA; ${ }^{6}$ College of Medicine, Department of Preventive and Social \\ Medicine, Charles R. Drew University of Medicine and Science, Los Angeles, CA, USA; ${ }^{7}$ David Geffen \\ School of Medicine, University of California, Los Angeles, Los Angeles, CA, USA; ${ }^{8}$ Department of \\ Medicine, Northwestern University Feinberg School of Medicine, Chicago, IL, USA
}

Background: Despite efforts by the AIDS Clinical Trials Group (ACTG) to enroll representative numbers of diverse women, participation in ACTG studies in the United States remains largely white and male. To address this gap in women's participation in ACTG research, a one-year pilot study of dedicated women's outreach workers (WOWs) was proposed.

Objectives: included demonstrating that targeted recruitment efforts can expand community awareness of ACTG research and ensuring successful enrollment of women at the respective clinical research sites.

Methods: The pilot study was conducted at two U.S. sites (Rutgers New Jersey Medical School and Emory Ponce de Leon Center in Atlanta, Georgia). The WOWs worked with site personnel to identify and reach out to women living with HIV and/or Hepatitis B or $\mathrm{C}$ at their respective sites and encourage them to join a clinical trial registry for those interested in participating in future clinical trials.

Results: The Rutgers WOW approached 127 potential participants (of whom 100 joined the WOW registry) and screened 35 participants for open ACTG studies. The Emory WOW approached 120 participants, enrolling 86 into the WOW registry, and screened 51 potential participants for open ACTG studies during the WOW's tenure. The majority of women screened at both sites were women of color.

Conclusions: The WOW study team identified several lessons learned that can inform future efforts to engage women living with HIV in clinical research. First, success in engaging women is proportional to level of funding and institutional support. Second, there is a need for a more gender-inclusive scientific agenda as women are more likely to participate if studies address topics of interest to them. Third, meaningful engagement is a two-way street.

KEYWORDS: HIV treatment trials, women, outreach workers, recruitment, clinical trials, HIV/AIDS, community engagement, gender

\section{Introduction}

Globally, the burden of HIV is disproportionately borne by women - cisgender and transgender - as over half of new HIV diagnoses are among women. Young women aged 15-24 years account for $80 \%$ of new HIV diagnoses in sub-Saharan Africa, and gender-based violence has been shown to increase the risk of HIV acquisition

Correspondence to: Elizabeth Barr National Institutes of Health, Office of Research on Women's Health, 6707 Democracy Boulevard, Suite 400, Bethesda, MD 20817, USA. Email: elizabeth.barr@nih.gov by as much as $150 \% .{ }^{1}$ In the U.S., $19 \%$ of new HIV diagnoses are in women, with significant variation by race, ethnicity, and geography., ${ }^{2,3}$ Enrolling women in HIV studies is a scientific and ethical imperative, particularly considering known sex differences in HIV infection, ${ }^{4}$ curative approaches, ${ }^{5}$ and outcomes. ${ }^{6}$ Yet, women are consistently under-enrolled in HIV research: a recent meta-analysis of gender differences in ART efficacy outcomes found that women represented $20 \%$ of participants in all randomized control trials for 
treatment of HIV infection submitted to the U.S. Food and Drug Administrative (FDA) for a New Drug Application (NDA) between 2000 and 2008, with the proportion of women declining over time. ${ }^{7,8}$ Women's under-enrollment is not unique to HIV and has been well-documented in multiple areas of biomedical research. $^{9-12}$

While a detailed exploration of the mechanisms underlying women's under-enrollment is outside of the scope of this paper, a number of reasons for women's under-enrollment in HIV trials have been posited, including clinical trials' contraceptive requirements, ${ }^{13}$ strict eligibility criteria that favor men, ${ }^{14}$ women's competing work, family, and childcare obligations, structural barriers (including stigma and systemic racism), ${ }^{15}$ and paternalistic attitudes from providers, pharmaceutical companies, and policymakers. ${ }^{16}$ Additionally, clinical research taking place today must contend with legacies of racial exploitation including medical mistrust (e.g., the U.S. Public Health Service (USPHS) Syphilis Study at Tuskegee or the case of Henrietta Lacks). ${ }^{17,18}$ Although the USPHS Syphilis Study officially ended in 1972 , the resulting medical mistrust remains visibly present in communities of color, particularly African American and Hispanic communities.

The federally-funded AIDS Clinical Trials Group (ACTG) is one of the world's largest HIV-related research networks with a mission of curing HIV and reducing the burden of disease due to HIV infection and its complications, including tuberculosis and viral hepatitis. ${ }^{19}$ The ACTG's Women's Health Inter-Network Scientific Committee (WHISC) helps ensure that the ACTG conducts clinical trials that specifically address the unique needs of women. The ACTG also benefits from an intentional, robust system of community involvement through its Global Community Advisory Board (GCAB), a network of local Community Advisory Boards (CABs), and a Community Scientific Subcommittee (CSS). Although the ACTG has made commendable efforts to enroll representative numbers of women and minority populations since its inception, participants in ACTG studies in the United States are predominantly white cisgender males. As of March 18, 2020, the network had completed 588 studies and enrolled over 132,922 participants worldwide. ${ }^{1}$ Just under $25 \% \quad(\mathrm{~N}=32,484 ; 24.4 \%)$ of all ACTG

\footnotetext{
'This number includes studies managed while the ACTG and International Maternal Pediatric Adolescent AIDS Clinical Trials (IMPAACT) Network were combined, and with participants in main and substudies counted separately. The numbers also include studies that were only open to female participants as well as studies that enrolled outside of the United States, where female enrollment tends to be higher.
}

participants have been female. ${ }^{2}$ A 2019 analysis of ACTG screening and enrollment in the U.S. between 2003-2013 found no significant sex difference in rates of protocol screening failures - when women were asked to participate in ACTG trials, women did not screen-out significantly more often than men. ${ }^{20}$

A consequence of women's under-inclusion in ACTG studies has been a sex and gender disparity that has slowed knowledge development of how to best treat women living with HIV, with a potential for lack of generalizability of scientific findings for women living with HIV. To address the gap in women's participation in ACTG research, a one-year, exploratory pilot study of dedicated women's outreach workers (WOWs) was proposed by a team that included researchers and community representatives. The primary objectives of the pilot study were to 1) demonstrate that targeted recruitment efforts can expand community awareness of ACTG research, 2) ensure successful enrollment of women at the respective clinical research sites, 3) strengthen links between sites and affected communities, 4) improve the health of women by engaging them in care, and 5) increase community understanding of and investment in the clinical research process. Secondary objectives were to create a registry of women who would be interested in being contacted for ACTG studies now or in the future and to create a women's outreach video for ACTG sites to aid in recruitment efforts. ${ }^{21}$

\section{Materials and methods}

The WOW pilot study was a community - investigator collaboration from conception through implementation. The study team included two ACTG community members and investigators from the ACTG's WHISC and Underrepresented Populations Committee (UPC). The WOW pilot study was proposed for full-time outreach workers at four ACTG sites. However, given across the board reductions in ACTG funding at the time, the WOW pilot study was awarded at $25 \%$ of the requested level. The WOW study team received a onemonth notice of the reduced award, and funds were required to be spent within nine months. The WOW study team decided to proceed forward opting to support half-time WOWs at two interested sites (Rutgers New Jersey Medical School and Emory Ponce de Leon Center in Atlanta, Georgia). The WOW pilot sites were chosen because they had a relatively large population of women living with HIV being cared for locally, a track record of being able to enroll

\footnotetext{
${ }^{2}$ Including both ACTG and IMPAACT studies, and with participants in main and substudies counted separately.
} 
women - particularly women of color - in clinical trials and had expressed interest in participating in the intervention. Both pilot sites were co-located with clinics that offer routine HIV care as well as research. Therefore, the sites had access to women who would be potentially eligible to participate in ongoing or planned ACTG studies. This pilot study was conducted between March 2016 and December 2017. Appendix A describes the ACTG studies that were open at each site during the WOW study period.

To ensure successful enrollment of women at the respective clinical research sites, WOWs worked with other site personnel to identify women living with HIV and/or Hepatitis $\mathrm{B}$ or $\mathrm{C}$ at their respective sites through review of clinic databases, chart review, referrals from providers and self-referrals. Women who agreed to participate in the WOW pilot had their medical records reviewed by the WOW. Data were entered in site-specific databases that allowed study staff to determine if women were eligible to participate in any enrolling ACTG clinical studies. The database also established a foundation for a registry that study staff could use to identify potential participants for future ACTG studies.

To strengthen links between sites and affected communities, WOWs reached out to medical clinics within their hospital setting and to women who were being seen at off-site locations: community-based organizations, community advisory boards (CABs), local food pantries, and women's shelters.

\section{Rutgers New Jersey Medical School WOW}

The WOW at Rutgers University had originally been hired as a study coordinator by the site in March 2016. The WOW pilot was approved by the Rutgers' Institutional Review Board (IRB) prior to implementation of the study (December 2016), and continued through December 2017. Upon approval of the pilot, the WOW was tasked with tracking the number of women who agreed to participate in the WOW project and to be included in the WOW registry database. The value of a potential participant registry was recognized early on in the WOW pilot program, leading this site to expand the protocol to also include men in their registry of future participants. The primary focus of the WOW program remained engaging and ultimately recruiting women in ACTG studies. The WOW also tracked the number of women who enrolled in open ACTG clinical studies and the number who agreed to join the registry as a result of being approached during this pilot.

The Rutgers WOW approached potential participants in the waiting room of the Rutgers Infectious Diseases Practice, primary care clinics, and by attending the many patient support groups conducted at the institution. IRB-approved flyers were posted in the waiting room of the clinics so interested study candidates could contact the WOW directly. The WOW also coordinated with the multi-disciplinary staff working in the Rutgers Infectious Diseases Practice such as case managers, social workers and mental health clinicians.

\section{Emory University WOW}

The Emory WOW started in September 2016 and assisted in recruiting women into ACTG clinical trials through November 2017. Emory study personnel operated under their ACTG site and other ongoing projects' IRB approvals, including a prescreening approval process. The primary focus of Emory's WOW outreach was engaging with and ultimately recruiting women in ACTG studies, although men were also approached for their potential participant registry. The WOW kept track of the number of women who enrolled in open ACTG clinical studies and those who were interested in enrolling into future studies.

Emory's WOW presented at women-specific support groups and Community Advisory Boards, including Emory Midtown Hospital, The Grady Infectious Disease Program, SisterLove and the Women's Interagency HIV Study (WIHS) community advisory board to increase awareness of HIV clinical trials. The Emory WOW also attended monthly meetings at the Emory Women's Clinic, made educational presentations to health and nursing classes at local universities and colleges, and hosted a table about clinical trial participation at seven local conferences and events.

\section{Results}

Although the longer timeline and larger budget originally proposed would have allowed the study team to develop, deploy, and test standardized WOW activities, the pilot enabled the two half-time WOWs to establish outreach programs using shared resources such as the women's outreach video and other pooled patient information sheets on available studies and ACTG recruitment information.

\section{Rutgers New Jersey Medical School WOW WOW registry}

From December 2016 - December 2017, the Rutgers WOW approached 127 potential participants for the WOW registry, 100 of whom entered the WOW registry. Participant demographics are shown in Table 1. Forty-five percent of participants on the Rutgers WOW registry were women, most of whom were Black (83\%) or Hispanic (9\%). Most women (95\%) were living with HIV; $12 \%$ had HCV infection (only $2 \%$ had HCV mono-infection); and 6\% had HBV/HIV 
Table 1. Demographics of participants enrolled in WOW registry.

\begin{tabular}{lcc}
\hline & Rutgers University (\%) & Emory University (\%) \\
\hline$N$ & 100 & 86 \\
Sex/Gender & & $46(53 \%)$ \\
Male & $55(55 \%)$ & $38(44 \%)$ \\
Female & $45(45 \%)$ & $2(2 \%)$ \\
Transgender women & $0(0 \%)$ & $9(11 \%)$ \\
Race/Ethnicity & $8(8 \%)$ & $67(85 \%)$ \\
White, Non-Hispanic & $83(83 \%)$ & $3(4 \%)$ \\
Black or African American, Non-Hispanic & $9(9 \%)$ & $86(100 \%)$ \\
Hispanic & & $4(5 \%)$ \\
Diagnoses & $95(95 \%)$ & $8(9 \%)$ \\
HIV & $6(6 \%)$ & $12(12 \%)$ \\
HBV & &
\end{tabular}
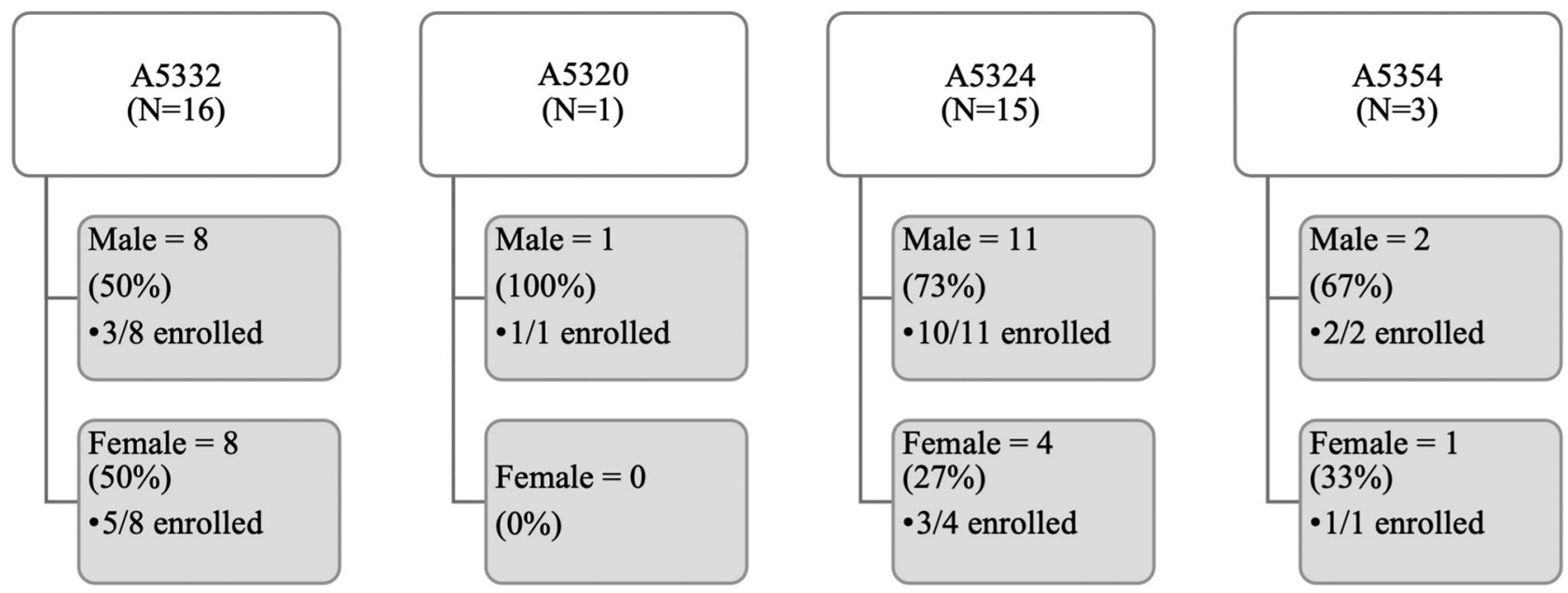

Figure 1. Potential participants screened and enrolled (Rutgers).

co-infection (none had HBV mono-infection). One female participant was unaware of her HIV, HBV or HCV status. Twenty-two of the 100 participants on the Rutgers registry had previously enrolled in ACTG studies. Of the remaining 78 participants, 14 were referred to open ACTG studies, and 3 and 2, respectively, enrolled in the REPRIEVE (A5332) and InMIND (A5324) ACTG studies. The other 9 participants referred to ACTG studies were found ineligible for the currently enrolling ACTG studies at their site.

The Rutgers WOW identified the following factors as key to improving women's enrollment: allowing staff time to build relationships with potential participants; meeting with women both individually and in group settings; providing support with childcare; and offering services and referrals intended to address the social inequalities experienced by women living with HIV (e.g., stigma, gender-based violence, financial insecurity). ${ }^{22,23}$

\section{ACTG studies}

In 2017, at Rutgers New Jersey Medical School four ACTG studies were actively enrolling. Appendix A provides brief descriptions of all ACTG studies described in this paper. The Rutgers site formally screened 35 potential participants for these studies during the WOW's tenure. Figure 1 depicts sex and enrollment outcomes for those participants who were captured in the ACTG's central database using the network screening system. The screening data reflected in Figure 1 does not include informal screenings (e.g., preliminary conversations with a potential participant) or chart reviews - only participants who provided consent for an ACTG study and were assigned a screening number. On average, $37 \%$ of participants screened were female, although sex of potential participants screened varied across studies (range $=0 \%-50 \%$ ).

\section{Emory University WOW WOW registry}

The Emory site approached 120 participants and enrolled 86 into the WOW registry between September 2016 and November 2017. Participant demographics are shown in Table 1. Forty-four percent of participants on the WOW registry were women, and the majority of participants were Black or African 

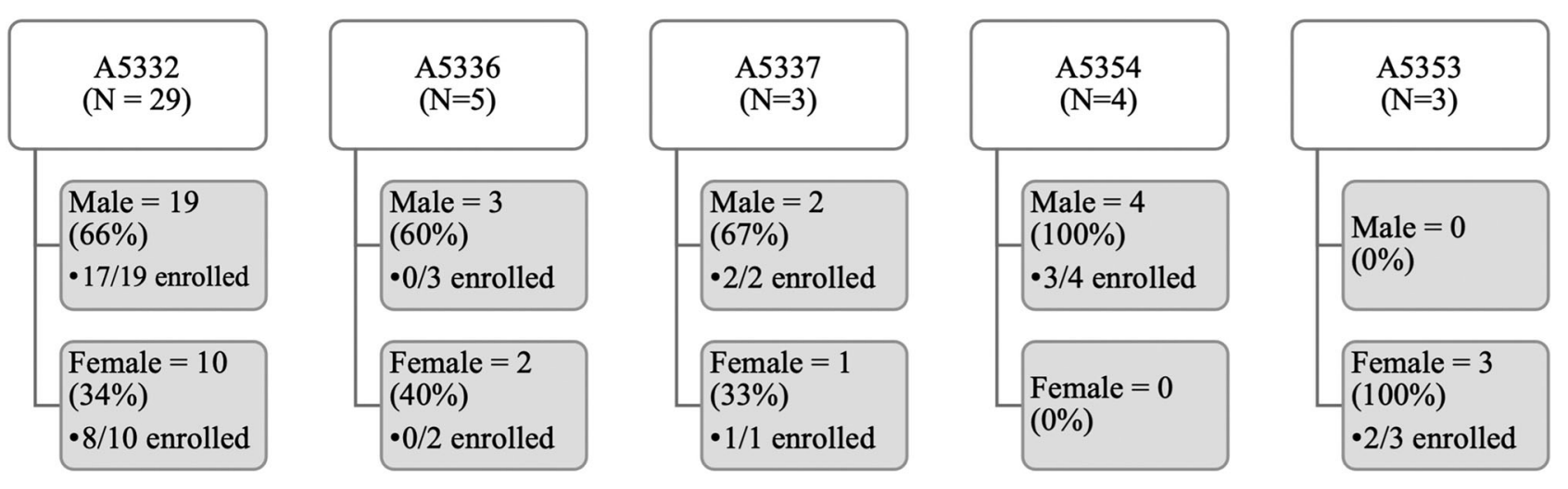

Figure 2. Potential participants screened and enrolled (Emory).

American (85\%). Two participants were transgender women. The Emory WOW identified the following factors as key to increasing women's enrollment: protocol-related budget for advertisement, marketing, recruitment events, and trinkets; shorter protocol time commitment; fewer invasive procedures; and liberal contraceptive requirements.

ACTG Studies. During the period the WOW was active, five ACTG studies were enrolling at Emory. These studies are described in Appendix A. Informal screenings, pre-screenings, and chart reviews identified 274 potential participants at Emory during the WOW period. Fifty-one of those participants were formally screened (provided consent and were assigned a screening number) for ACTG studies during this time. Figure 2 shows formal screenings and subsequent enrollments, broken down by sex. Just over $40 \%$ of those formally screened were female, although variation existed across studies (range $=0 \%-100 \%$ ).

\section{Discussion and lessons learned}

As proof of concept, a one-year, $50 \%$ effort of women's outreach workers was not ideal for building the long-term relationships required to increase community engagement, nor for collecting the longitudinal data required to evaluate engagement. Enrolling women in clinical trials-HIV-related or not-often requires efforts different than those traditionally effective at recruiting men, ${ }^{24-29}$ but ACTG sites had rarely been given resources to hire and train personnel such as outreach workers to devote to this mission. Recently, individual ACTG studies have made additional outreach funding available to sites, although this is not yet standard practice across all ACTG studies. It has been well-documented that women are more likely to participate in clinical trials when the research institution builds a long-term, trusting, reciprocal relationship that acknowledges the multidimensionality of women's lives. ${ }^{30-33}$

Despite obstacles encountered in implementing this exploratory pilot, the WOW study team was able to identify several lessons learned that can inform future efforts to engage women living with HIV in clinical research. ACTG leadership remained supportive of this pilot study, inviting the WOW team to share their results at the annual ACTG meetings as part of ongoing conversations on strategies to increase enrollment of women in ACTG studies. The lessons learned during the WOW pilot are informed by the study team's previous work within the ACTG and discussions with community advocates, investigators, study staff and study participants. Table 2 summarizes the lessons learned from the WOW pilot program implementation.

\section{Lesson 1: Success in engaging women requires funding and institutional support}

The WOW pilot study demonstrated that it is possible to identify staff to devote $50 \%$ time to increase the enrollment of women in ACTG trials into their HIV clinical trials unit - when funding and institutional support are available. The ACTG has a stated commitment to both ensuring its priorities are in alignment with affected communities and enrolling diverse individuals in studies. Individual sites demonstrate creativity and commitment to enrolling diverse women. However, the success of women's enrollment initiatives will depend on both the level of funding and perceived institutional support of the effort. Consistent, ongoing, dedicated enrollment funds at both the site and/or study level would facilitate the fulfillment of these commitments. Further, enrollment-focused community engagement activities should include more than current site $\mathrm{CAB}$ members and extend beyond the site's infectious diseases clinic to obtain maximum effectiveness. One intention of community engagement 
Table 2. Lessons learned from wOW pilot program aimed at increasing enrollment of women in ACTG clinical trials (2016 - 2017).

\begin{tabular}{|c|c|}
\hline Lessons & Implications \\
\hline $\begin{array}{l}\text { Lesson 1: Success in engaging women is proportional to level } \\
\text { of funding and institutional support }\end{array}$ & $\begin{array}{l}\text { - It is possible to have a dedicated effort to increase the } \\
\text { enrollment of women in ACTG trials, especially when funding } \\
\text { and institutional support is available for that effort. } \\
\text { - Separate lines of funding can ensure that activities are } \\
\text { appropriately staffed and maintained. }\end{array}$ \\
\hline Lesson 2: Need for a gender-inclusive scientific agenda & $\begin{array}{l}\text { - The network's scientific agenda must reflect the social } \\
\text { determinants of health that impact multiply } \\
\text { marginalized women. } \\
\text { - Prioritizing gender-inclusivity in all ACTG studies would allow } \\
\text { the network's researchers to answer the scientific questions } \\
\text { that drive ACTG research in ways that are relevant for all } \\
\text { people affected by HIV. }\end{array}$ \\
\hline Lesson 3: Meaningful engagement is a two-way street & $\begin{array}{l}\text { - Engagement includes bringing community members "to the } \\
\text { table" on CABs and research teams as well as going "into } \\
\text { the field." } \\
\text { - Research participants value referrals and other social } \\
\text { services resources. }\end{array}$ \\
\hline
\end{tabular}

and outreach is to reach - and hopefully enroll - new participants who have never participated in clinical studies. Outreach can open doors to women living with HIV who may not be currently active in care or who have never thought to participate in a clinical trial. Forging these new contacts and connections requires dedicated, ongoing time and effort.

\section{Lesson 2: a gender-inclusive scientific agenda accounts for sex and gender and responds to social and behavioral factors}

A gender-inclusive scientific agenda can facilitate the inclusion of questions relevant to women across the ACTG network. A gender-inclusive women's HIV research agenda will consider biological factors such as sex differences in HIV infection, ${ }^{4}$ explore drug-drug interactions between hormone therapy and ART, ${ }^{34}$ and examine structural and cultural factors such as stigma ${ }^{35}$ and sexual violence ${ }^{36}$ as rigorously as biomedical factors. As one example, the scientific objectives of ACTG A5375 (a pharmacokinetic study assessing dose adjustments of levonorgestrel emergency contraception when used in combination with ART or anti-tuberculosis (TB) therapy) are in part informed by the high prevalence of sexual assault among women living with HIV $^{37-39}$ and the World Health Organization's recommendations for provision of emergency contraception after sexual assault. ${ }^{40}$

Both the Rutgers and Emory WOWs experienced success with recruiting women into the WOW registry, providing further evidence that women do want to participate in trials - when explicitly asked. However, if there are few studies that specifically address women's health needs, there may be few studies that women are interested in joining. The MOXIE Trial for post-menopausal women (ACTG A5366) was the first women-only
HIV cure-related clinical trial, testing a latency-reversal agent. The MOXIE Trial opened after the WOW project was completed and was one of the fastest enrolling studies in the ACTG, demonstrating that not only is it possible to enroll women in ACTG studies, but that women appreciate having studies that answer questions specific to their health. ${ }^{41}$

Importantly, while A5375 and The MOXIE Trial are examples of studies that enrolled only females, gender-inclusivity does not mean female-exclusivity. Prioritizing gender-inclusivity in all ACTG studies would allow the network's researchers to answer scientific questions that drive ACTG research in ways that are relevant for all people affected by HIV. Efforts undertaken to enroll women can also be applicable to men - experiences of stigma, violence, housing insecurity, or incarceration are by no means unique to women. Recognizing and responding to these social factors through a gender-inclusive agenda can "promote rigorous, reproducible and responsible science." $"$ Integration of socio-behavioral research as part of clinical trials can also provide an increased understanding of what those social factors are and how clinical trial teams can best be responsive to participants' needs. ${ }^{43}$

\section{Lesson 3: Meaningful engagement is a two- way street}

Ongoing and sustained community collaborations and partnerships have been shown to foster women's willingness to participate in HIV research. ${ }^{31,44,45}$ The WOW study team prioritized community perspectives: a GCAB member (EB) was one of the proposing investigators for the WOW pilot, and two GCAB members (EB \& DC) were involved in study implementation and analysis. Creating space for community 
voices within the structure of the ACTG ensures women living with and affected by HIV have opportunities to shape research into these complex factors. Meaningful engagement with women also entails a recognition of the intersecting factors that shape diverse women's lives, including gender, race, poverty, and other social determinants of health. ${ }^{46-49}$ Attention to structural level drivers of health inequity is a key element of patient-centered-care ${ }^{50,51}$ and a critical component of meaningful community engagement. ${ }^{52,53}$

Anecdotal evidence suggests that study personnel are sometimes reluctant to enroll women whom they perceive as potentially unable to complete the study and likely to leave the study prematurely which would reflect poorly on the enrolling clinical site. A 2007 survey of ACTG study coordinators and nurses found that women, people of color, and people with a history of injection drug use were perceived as less interested in clinical research - a perception that seems likely to influence study personnel's recruitment decisions. ${ }^{54}$ Women of color, transwomen, and members of other marginalized communities are aware of this provider referral bias and stigma, and perceive it as a barrier to engagement in HIV care and research. ${ }^{55,56}$ Staff trainings on cultural sensitivity, like those developed by the DAIDS Transgender Working Group, may be able to address this enrollment barrier. ${ }^{57}$

The ACTG's CAB structure is an important mechanism through which ACTG researchers reach affected communities. Early and ongoing community engagement involves simultaneously bringing community members "to the table" through processes like local CABs, and going "into the field" to raise awareness of HIV clinical trials in the community. Both the Rutgers and Emory sites documented that many of the women contacted by the WOW had social and health needs that extended beyond trial participation: food, housing, social services/ case management, mental health services, and detoxication services. Offering supportive social services and referrals can go a long way towards transforming research sites from a place of historical racialized exploitation $^{58}$ and (re)building trust and trustworthiness in medical establishments and research programs. ${ }^{31}$

\section{Limitations and future directions}

This pilot of a pilot had two notable limitations: the sites selected were not necessarily representative of all U.S. ACTG sites, and the study was scaled back from four fulltime WOWs to two 50\%-time WOWs. However, some site-level successes can be replicated elsewhere. For example, identifying staff who can serve as unofficial community liaisons and offering informal education or referrals to care can foster trust and comfort for potential participants and their providers. Connecting women living with HIV to case managers who can help address their social service needs further helps women living with HIV feel supported and engenders trust with the healthcare system.

This exploratory study underscores the importance of understanding women's motivations for participation in ACTG trials and reveals several areas for future research, including focus groups and sustained discussions with women living with HIV. Region-specific focus groups and socio-behavioral science research could capture potential participants' perceptions on and experience with clinical trials - why women want to participate in research or not - as well as identify factors that enable participation. These participant-centered data would allow individual sites to tailor their efforts to better serve their unique communities of women. Results of that research would also inform the ACTG's scientific agenda and priorities - what studies to pursue - and influence the ACTG's future financial and policy decisions.

\section{Acknowledgments}

The authors acknowledge the study coordinators at the Rutgers and Emory sites, including Baljinder Singh (Rutgers) and Anna Benbrook (Emory). We also acknowledge the support of Michelle Cespedes and Jose Castillo-Mancilla of the ACTG's UPC, Cindy Firnhaber and Elizabeth Connick of the ACTG's WHISC, and Kris Coughlin, the ACTG's chief data manager. We are grateful for the valuable input Catherine Godfrey, Sarah Looby and Aadia Rana provided during the development and implementation of this pilot. We acknowledge the support of ACTG leadership, including Judith Currier and Daniel Kuritzkes and are appreciative of the generative feedback provided by the editor and anonymous reviewers.

\section{Disclosure statement}

The authors report no conflicts of interest.

\section{Disclaimer}

This article was prepared while Elizabeth Barr was affiliated with the ACTG CSS. The opinions expressed in this article are the author's own and do not reflect the view of the National Institutes of Health, the Department of Health and Human Services, or the United States Government. 


\section{Funding}

Research reported in this publication was supported by the National Institute of Allergy and Infectious Diseases of the National Institutes of Health under Award Number UM1 AI068634, U01AI69471, UM1 AI069418-08, UM1 AI069419-08, UM1 AI068636 and UM1 AI106701. The content is solely the responsibility of the authors and does not necessarily represent the official views of the National Institutes of Health.

\section{ORCID}

Elizabeth Barr (D) http://orcid.org/0000-00026115-1377

Karine Dubé (D) http://orcid.org/0000-0003-3458-1539

Carlos Del Rio (D) http://orcid.org/0000-0002-

0153-3517

\section{References}

1 UNAIDS. Global HIV \& AIDS statistics - 2020 fact sheet. 2020.

2 Hodder SL, Justman J, Hughes JP, et al. HIV acquisition among women from selected areas of the United States: a cohort study. Ann Intern Med. 2013;158(1):10-18.

3 Centers for Disease Control and Prevention. Diagnoses of HIV infection in the United States and dependent areas, 2018 (updated). HIV Surveillance Report. 2020;31doi:http://www.cdc. gov/hiv/library/reports/hiv-surveillance.html

4 Scully EP. Sex Differences in HIV Infection. Curr HIV/AIDS Rep. 2018;15(2):136-146.

5 Gianella S, Tsibris A, Barr L, Godfrey C. Barriers to a cure for HIV in women. J Int AIDS Soc. 2016;19(1):20706.20706

6 Cohn J, Ake J, Moorhouse M, Godfrey C. Sex Differences in the Treatment of HIV. Curr HIV/AIDS Rep. 2020;17(4):373-384.

7 Soon GG, Min M, Struble KA, et al. Meta-analysis of gender differences in efficacy outcomes for HIV-positive subjects in randomized controlled clinical trials of antiretroviral therapy (2000-2008). AIDS Patient Care STDS. 2012;26(8):444-453.

8 d'Arminio Monforte A, González L, Haberl A, Sherr L, SsanyuSseruma W, Walmsley SL. Better mind the gap: addressing the shortage of HIV-positive women in clinical trials. Aids. 2010; 24(8):1091-1094

9 Gong IY, Tan NS, Ali SH, et al. Temporal trends of women enrollment in major cardiovascular randomized clinical trials. Can J Cardiol. 2019;35(5):653-660.

10 Khan SU, Khan MZ, Raghu Subramanian C, et al. Participation of women and older participants in randomized clinical trials of lipid-lowering therapies: a systematic review. JAMA Netw Open. 2020;3(5):e205202.

11 Kwiatkowski K, Coe K, Bailar JC, Swanson GM. Inclusion of minorities and women in cancer clinical trials, a decade later: Have we improved? Cancer 2013;119(16):2956-2963.

12 Curno MJ, Rossi S, Hodges-Mameletzis I, Johnston R, Price MA, Heidari S. A systematic review of the inclusion (or exclusion) of women in HIV research: from clinical studies of antiretrovirals and vaccines to cure strategies. J Acquir Immune Defic Syndr. 2016;71(2):181-188.

13 Sullivan KA, Little MO, Rosenberg NE, et al. Women's views about contraception requirements for biomedical research participation. PLoS One. 2019;14(5):e0216332.

14 Gandhi M, Ameli N, Bacchetti P, et al. Eligibility criteria for HIV clinical trials and generalizability of results: the gap between published reports and study protocols. Aids. 2005; 19(16):1885-1896.

15 Fabian KE, Huh D, Kemp CG, et al. Moderating Factors in an Anti-stigma Intervention for African American Women with HIV in the United States: A Secondary Analysis of the UNITY Trial. AIDS Behav. 2019;23(9):2432-2442.
16 Gollub EL. Human rights is a US problem, too: the case of women and HIV. Am J Public Health. 1999;89(10):1479-1482.

17 Jones BL, Vyhlidal CA, Bradley-Ewing A, Sherman A, Goggin $\mathrm{K}$. If we would only ask: how Henrietta lacks continues to teach us about perceptions of research and genetic research among African Americans today. J Racial Ethn Health Disparities. 2017;4(4):735-745.

18 Brincks AM, Shiu-Yee K, Metsch LR, et al. Physician mistrust, medical system mistrust, and perceived discrimination: associations with HIV care engagement and viral load. AIDS Behav. 2019;23(10):2859-2869.

19 ACTG Network. About the ACTG. https://actgnetwork.org/ about-the-actg/

20 Smeaton LM, Kacanek D, Mykhalchenko K, et al. Screening and enrollment by sex in human immunodeficiency virus clinical trials in the United States. Clin Infect Dis 2020;71(5):1300-1305.

21 ACTG Network. Understanding the importance of women and minorities participating in ACTG network clinical trials. 2016. https://www.youtube.com/watch?v=jmaaMv1PalA

22 Kalichman S, Shkembi B, Hernandez D, Katner H, Thorson KR. Income inequality, HIV stigma, and preventing HIV disease progression in rural communities. Prev Sci. 2019;20(7):1066-1073.

23 Willie TC, Stockman JK, Perler R, Kershaw TS. Associations between intimate partner violence, violence-related policies, and HIV diagnosis rate among women in the United States. Ann Epidemiol. 2018;28(12):881-885.

24 Goff SL, Youssef Y, Pekow PS, et al. Successful strategies for practice-based recruitment of racial and ethnic minority pregnant women in a randomized controlled trial: the IDEAS for a healthy baby study. J Racial and Ethnic Health Disparities. 2016;3(4):731-737.

25 Peindl KS, Wisner KL. Successful recruitment strategies for women in postpartum mental health trials. J Psychiatr Res. 2003;37(2):117-125

26 Aguirre TM, Koehler AE, Joshi A, Wilhelm SL. Recruitment and retention challenges and successes. Ethn Health. 2018;23(1): 111-119.

27 King DW, Duello TM, Miranda PY, et al. Strategies for recruitment of healthy premenopausal women into the African American Nutrition for Life (A NULIFE) study. $J$ Womens Health (Larchmt). 2010;19(5):855-862.

28 Braun LA, Kennedy HP, Sadler LS, Dixon J, Research On US. Research on U.S. military women: recruitment and retention challenges and strategies. Mil Med. 2015;180(12):1247-1255.

29 Leonard A, Hutchesson M, Patterson A, Chalmers K, Collins C. Recruitment and retention of young women into nutrition research studies: practical considerations. Trials 2014;15(1):23.

30 Webster K, Carter A, Proulx-Boucher K, et al. Strategies for recruiting women living with human immunodeficiency virus in community-based research: lessons from Canada. Prog Community Health Partnersh. 2018;12(1):21-34.

31 Loutfy MR, V LK, Mohammed S, et al. Recruitment of HIVpositive women in research: discussing barriers, facilitators, and research personnel's knowledge. TOAIDJ 2014;8(1):58-65.

32 Falcon R, Bridge DA, Currier J, et al. Recruitment and retention of diverse populations in antiretroviral clinical trials: practical applications from the gender, race and clinical experience study. $J$ Womens Health (Larchmt). 2011;20(7):1043-1050.

33 DeMarco RF, Lanier LR. The concept of "silencing the self" in low-income, aging, HIV-infected African American women: a 10-year community-based participatory program of research with results. J Assoc Nurses AIDS Care. 2014;25(2):112-122.

34 Radix A, Sevelius J, Deutsch MB. Transgender women, hormonal therapy and HIV treatment: a comprehensive review of the literature and recommendations for best practices. $J$ Int AIDS Soc. 2016;19(3 Suppl 2):20810.20810

35 Kemp CG, Lipira L, Huh D, et al. HIV stigma and viral load among African-American women receiving treatment for HIV. Aids. 2019;33(9):1511-1519.

36 Aldous A, Magnus M, Roberts A, et al. Challenges in conducting research on sexual violence and HIV and approaches to overcome them. Am J Reprod Immunol. 2017;78(1):e12699.

37 Smith LR, Yore J, Triplett DP, et al. Impact of Sexual Violence Across the Lifespan on HIV Risk Behaviors Among Transgender Women and Cisgender People Living With HIV. J Acquir Immune Defic Syndr. 2017;75(4):408-416. 
38 Tenkorang EY, Asamoah-Boaheng M, Owusu AY. Intimate Partner Violence (IPV) Against HIV-Positive Women in Sub-Saharan Africa: A Mixed-Method Systematic Review and Meta-Analysis. Trauma Violence Abuse. Advance online publication. 2020.

39 Harrison SE, Brown M, Cho H. Links between sexual trauma exposure and Quality of Life (QoL) domains among people living with HIV in the Southern United States. AIDS Care. 2020; 32(sup2):91-98.

40 World Health Organization. Emergency contraception. 2018.

41 Dubé K, Hosey L, Starr K, et al. Participant perspectives in an HIV cure-related trial conducted exclusively in women in the United States: results from AIDS clinical trials group 5366. AIDS Res Hum Retroviruses. 2020;36(4):268-282.

42 Tannenbaum C, Schwarz JM, Clayton JA, de Vries GJ, Sullivan C. Evaluating sex as a biological variable in preclinical research: the devil in the details. Biol Sex Differ. 2016;7:13.

43 Dubé K, Barr L, Palm D, Brown B, Taylor J. Putting participants at the centre of HIV cure research. Lancet HIV. 2019;6(3): e147-e149.

44 Kaida A, Carter A, Nicholson V, et al. Hiring, training, and supporting peer research associates: operationalizing communitybased research principles within epidemiological studies by, with, and for women living with HIV. Harm Reduct J. 2019;16(1):47.

45 Kennedy T, Teti M, Hayes D, Pichon LC, Farnan R. Unmasked to a positive triumph": women with HIV share the benefits of showing their faces through photovoice. Prog Community Health Partnersh. 2016;10(4):505-513.

46 Rao D, Andrasik MP, Lipira L. HIV stigma among black women in the United States: intersectionality, support, resilience. $A m J$ Public Health. 2018;108(4):446-448.

47 McCree DH, Chesson H, Bradley ELP, Lima A, Fugerson AG. US regional changes in racial/ethnic disparities in HIV diagnoses among women in the United States, 2012 and 2017. AIDS Behav. 2020;24(4):1118-1123.

48 Reisner SL, Chaudhry A, Cooney E, Garrison-Desany H, JuarezChavez E, Wirtz AL. It all dials back to safety': A qualitative study of social and economic vulnerabilities among transgender women participating in HIV research in the USA. BMJ Open. 2020;10(1):e029852.
49 Erickson M, Shannon K, Sernick A, et al. Women, incarceration and HIV: a systematic review of HIV treatment access, continuity of care and health outcomes across incarceration trajectories. Aids. 2019;33(1):101-111.

50 Sullivan KA, Schultz K, Ramaiya M, Berger M, Parnell H, Quinlivan EB. Experiences of women of color with a nurse patient navigation program for linkage and engagement in HIV care. AIDS Patient Care STDS. 2015;29(S1):S49-S54.

51 Byrd KK, Hardnett F, Clay PG, et al. Retention in HIV care among participants in the patient-centered HIV care model: a collaboration between community-based pharmacists and primary medical providers. AIDS Patient Care STDS. 2019;33(2): 58-66.

52 Karris MY, Dubé K, Moore AA. What lessons it might teach us? Community engagement in HIV research. Curr Opin HIV Aids. 2020;15(2):142-149.

53 Lessard D, Engler K, Vicente S, Bilodeau M, Lebouché B. Challenges of patient engagement in an HIV clinical research program: a qualitative analysis of stakeholder accounts. J Patient Exp. 2020;7(6):925-930.

54 King WD, Defreitas D, Smith K, et al. Attitudes and perceptions of AIDS clinical trials group site coordinators on HIV clinical trial recruitment and retention: a descriptive study. AIDS Patient Care STDS. 2007;21(8):551-563.

55 Geter A, Sutton MY, Hubbard McCree D. Social and structural determinants of HIV treatment and care among black women living with HIV infection: a systematic review: 2005-2016. AIDS Care. 2018;30(4):409-416.

56 Erves JC, Mayo-Gamble TL, Malin-Fair A, et al. Needs, priorities, and recommendations for engaging underrepresented populations in clinical research: a community perspective. $J$ Commun. Health. 2017;42(3):472-480.

57 Siskind RL, Andrasik M, Karuna ST, et al. Engaging transgender people in NIH-funded HIV/AIDS clinical trials research. $J$ Acquir Immune Defic Syndr. 2016;72(3):S243-S7.

58 Jaiswal J, Singer SN, Siegel K, Lekas HM. HIV-related 'conspiracy beliefs': lived experiences of racism and socio-economic exclusion among people living with HIV in New York City. Cult Health Sex. 2019;21(4):373-386.

\section{Appendix A.}

\section{ACTG studies enrolling at sites with WOWs}

\begin{tabular}{llcc}
\hline Study Number & \multicolumn{1}{c}{ Brief Description } & $\begin{array}{c}\text { Enrolling at } \\
\text { Rutgers }\end{array}$ & $\begin{array}{c}\text { Enrolling at } \\
\text { Emory }\end{array}$ \\
\hline A5332 (REPRIEVE) & $\begin{array}{l}\text { Evaluates the role of a statin medication (pitavastatin calcium) in preventing } \\
\text { heart disease among people with HIV }\end{array}$ & Yes & Yes \\
A5320 & Viral Hepatitis C Infection Long-term Cohort Study & Yes & No \\
A5324 & Study of Maraviroc and Dolutegravir's impact on neurocognitive performance & Yes & No \\
A5336 & Phase II study of the safety of ruxolitinib (a kinase inhibitor) in virologically- & No & Yes \\
& suppressed, HIV-positive participants & No & Yes \\
A5337 & Phase I/II study to evaluate the safety of sirolimus (a chemotherapy drug) & No & Yes \\
A5353 & Phase II study of dolutegravir plus lamivudine as an initial regimen & Yes & Yes \\
A5354 & Study of early ART initiation in acute HIV infection; participants are required to & & \\
& have a diagnosis of acute HIV within 7 days prior to enrollment & \\
\hline
\end{tabular}

